CIENCIAS NATURALES Y EXACTAS

INVESTIGACIÓN

\title{
Efectos protectores de los alimentos andinos contra el daño producido por el alcohol a nivel del epitelio intestinal, una aproximación estadística
}

Heilbron, Eduardo*; Martin, Osvaldo*^ y Fumagalli, Emiliano*

\section{Resumen}

La barrera epitelial intestinal es altamente regulada y permite el pasaje selectivo de nutrientes, mientras que es impermeable a sustancias nocivas. Las uniones entre las células de este epitelio tienen estructuras proteicas que son responsables de mantener dicha impermeabilidad. Existen sustancias que pueden dañar la impermeabilidad de la barrera epitelial intestinal, como el etanol. Existen sustancias en los alimentos que aminoran el daño que puede sufrir la mencionada barrera. En este trabajo se buscó relacionar patrones de consumo de alcohol, la calidad de vida desde el punto de vista de la salud intestinal y la frecuencia de consumo de grupos de alimentos.

Palabras clave: consumo de alcohol; calidad de vida; alimentos andinos; sistema digestivo

El presente artículo es el resultado de un proyecto de investigación financiado por la Secretaría de Ciencia, Técnica y Estudios Regionales de la Universidad Nacional de Jujuy. Recibido el 16/03/2020 y aprobado el 04/09/2020.

DOI: https://doi.org/10.33255/3161/747

Autoría: ${ }^{*}$ Instituto de Estudios Celulares Genéticos y Moleculares, UNJu-Conicet, Argentina. ${ }^{*}$ Instituto de Matemática Aplicada (UNSL-Conicet), Argentina.

Contacto: eheilbron@conicet.gov.ar 
Protective effects of Andean foods against the damage produced by alcohol at the level of the intestinal epithelium, a statistical approximation

\begin{abstract}
The intestinal epithelial barrier is highly regulated and allows the selective passage of nutrients, while being impermeable to harmful substances. The junctions between the cells of this epithelium have protein structures that are responsible for maintaining this impermeability. There are substances that can damage the impermeability of the intestinal epithelial barrier, such as ethanol. There are substances in food that reduce the damage that this barrier can suffer. In this work, we sought to relate patterns of alcohol consumption, quality of life from the point of view of intestinal health and the frequency of consumption of food groups.
\end{abstract}

Keywords: alcohol consumption; quality of life; Andean foods; digestive system

\title{
Efeitos protetores dos alimentos andinos contra os danos produzidos pelo álcool ao nível do epitélio intestinal, uma aproximação estatística
}

\section{Resumo}

A barreira epitelial intestinal é altamente regulada e permite a passagem seletiva de nutrientes, sendo impermeável a substâncias nocivas. As junções entre as células desse epitélio possuem estruturas proteicas que são responsáveis por manter essa impermeabilidade. Existem substâncias que podem danificar a impermeabilidade da barreira epitelial intestinal, tais como o etanol. Existem substâncias nos alimentos que reduzem os danos que essa barreira pode sofrer. Neste trabalho, buscou-se relacionar padrões de consumo de álcool, qualidade de vida do ponto de vista da saúde intestinal e a frequência de consumo de grupos de alimentos.

Palavras chave: consumo de álcool; qualidade de vida; alimentos andinos; sistema digestivo 


\section{Introducción}

El intestino delgado posee una monocapa de células epiteliales responsable de mantener una barrera semipermeable, altamente regulada, de manera que permite el pasaje selectivo de nutrientes mientras que evita el ingreso de sustancias nocivas al torrente sanguíneo. Las células de este epitelio poseen uniones ocluyentes, que son las principales responsables de mantener la impermeabilidad de la monocapa (Salvo Romero et al., 2015). El daño a esta barrera permite el pasaje de microorganismos y sustancias nocivas que aumentan el riesgo de que eventualmente se desarrollen enfermedades inflamatorias intestinales, como la enfermedad de Crohn y la colitis ulcerosa (Anderson y Van Itallie, 2009). Asimismo, el ingreso de endotoxinas desde el lumen intestinal al torrente sanguíneo ha sido asociado al agravamiento de la enfermedad hepática alcohólica (Keshavarzian et al., 1999). El etanol es una de las sustancias que tienen un claro efecto nocivo para la barrera epitelial intestinal. Estudios in vivo e in vitro indican que el consumo de alcohol está asociado a un aumento de la permeabilidad intestinal (Pascual et al., 2001). La salud intestinal tiene una importante influencia en varios aspectos de la calidad de vida, incluyendo las dimensiones física, psicológica, familiar y social (Casellas et al., 2002; Vergara et al., 2002; Casellas et al., 2001).

Ciertos alimentos pueden tener beneficios para la salud intestinal por su aporte de prebióticos, probióticos o protegiendo las uniones ocluyentes. Los polifenoles, que en general tienen efectos antioxidantes (Quideau et al., 2011), muestran efectos protectores sobre la barrera epitelial intestinal (Yang et al., 2017). Por lo tanto, se espera que dietas que contengan alimentos ricos en polifenoles puedan asociarse a una mejor calidad de vida desde el punto de vista de la salud intestinal. A su vez, el consumo de alcohol estaría asociado a una peor calidad de vida desde el punto de vista de la salud intestinal. En este trabajo se analizan las relaciones entre la calidad de vida desde el punto de vista de la salud intestinal, los patrones de consumo de alcohol y la frecuencia de consumo de diferentes grupos de alimentos.

\section{Materiales y métodos}

Luego del análisis y firma de un consentimiento informado, se utilizaron tres cuestionarios autocompletados en una muestra total de 141 individuos de entre 18 y 65 años, de ámbitos urbanos de San Salvador de Jujuy. Para estimar el patrón de consumo de alcohol se utilizó el Cuestionario de Identificación de Trastornos Debidos al Consumo de Alcohol (AUDIT, en su versión en castellano) 
(Babor, T. et al., 2001). Este cuestionario desarrollado por la OMS consta de diez preguntas, agrupadas en tres tópicos: consumo peligroso de alcohol, dependencia al alcohol y consumo dañino de alcohol. El AUDIT es una herramienta ampliamente validada y posee una alta consistencia interna, con un $\alpha$ de Cronbach de 0,879 (Medina et al., 2015).

Para medir la calidad de vida desde el punto de vista de la salud intestinal se usó la versión en español del cuestionario de calidad de vida de 32 ítems de la enfermedad inflamatoria intestinal (IBDQ 32) (Guyatt et al., 1993). Las preguntas se orientan al análisis de cuatro dimensiones: dimensión digestiva, dimensión sistémica, dimensión emocional y dimensión social. Cada pregunta se responde con una escala tipo Likert puntuada de 1 (baja calidad de vida) a 7 (alta calidad de vida), con un rango de 32-224 puntos. Este cuestionario posee excelentes propiedades psicométricas de consistencia interna ( $\alpha$ de Cronbach superior a 0,9), validez (prueba del tamaño del efecto superior a 1,7 ) y fiabilidad (coeficiente de correlación intraclase superior a 0,8) (Masachs et al., 2007).

La frecuencia de consumo de diversos alimentos se estimó mediante el Cuestionario de Frecuencia de Alimentación (FFQ, Food Frecuency Questionnarie) (Willett et al., 1985) de 118 ítems que fue adaptado para este trabajo, al considerar once grupos alimenticios y al colocar quínoa, amaranto y papa andina como ítems en el cuestionario. El mismo fue originalmente desarrollado para poner a prueba hipótesis epidemiológicas y nutricionales. La reproducibilidad del mismo fue comparable a la observada en registros nutricionales, mostrando coeficientes de correlación que son considerados aceptables para este tipo de instrumento (entre $r=0,51$ y $r=0,88$ ) (Martin-Moreno et al., 1993).

Los datos fueron procesados usando Pandas (McKinney, 2011). Los gráficos, usando Matplotlib (Hunter, 2007) o ArviZ (Kumar et al., 2019) para los resultados de modelos bayesianos. Los análisis estadísticos se realizaron usando PyMC3 (Salvatier et al., 2016), una librería de Python para estadística bayesiana. Se realizó un análisis de las componentes principales entre las variables IBDQ32, AUDIT, consumo de papa andina mensual y consumo de quínoa y amaranto mensual. Para este análisis se utilizó la implementación de PCA de Scikit-learn (Pedregosa et al., 2011) que es un PCA probabilístico tal cual se describe en «Probabilistic Principal Component Analysis on JSTOR» (Tipping y Bishop, 1999). Se utilizaron los parámetros por defecto y los datos fueron estandarizados antes de hacer el PCA. 


\section{Resultados}

Para analizar el patrón de consumo de alcohol de las personas que participaron en el estudio se utilizó la encuesta AUDIT, en la que los valores más elevados indican un patrón consistente con daños producidos por el excesivo consumo de alcohol y posible adicción al mismo. AUDIT es una variable discreta que va entre 0 y 24 y su distribución puede ser aproximada por una distribución cero-inflada negativa binomial (datos no mostrados). Los puntajes obtenidos para la encuesta AUDIT muestran el $30,6 \%$ con un valor de cero, con una media de 3,69 puntos y una varianza de 1,10 (Figura 1A). El punto de corte desde el cual el consumo se considera riesgoso y puede generar problemas de salud, psicológicos o sociales, es un puntaje de AUDIT de 8 o más (Babor, T. et al., 2001). En nuestra muestra, eso representa el 13,6\% (19 casos). La media en las mujeres es más baja que en hombres (2,9 y 4,7, respectivamente). El consumo de alcohol disminuye con la edad; el mayor consumo se da en la franja etaria de 18 a 35 años en varones y en mujeres.

La calidad de vida desde el punto de vista de la salud intestinal se midió mediante la escala generada por el IBDQ32, la cual es una variable discreta que se distribuye en el rango de 32 a 224 (en nuestra muestra, entre 80 y 224). Evaluamos el ajuste de la muestra IBDQ32 a tres distribuciones: negativa binomial, normal y normal truncada (límite superior 224). Los valores del IBDQ32 tiene una media de 193,1 (y un valor máximo de 224) y una desviación estándar de 24,2 (Figura 1B). Usamos WAIC (Watanabe, 2013) a fin de evaluar el ajuste. Encontramos que la distribución con un valor de WAIC inferior (y por lo tanto el mejor modelo, entre las alternativas evaluadas) es una normal truncada (Figura 2). La dimensión gastrointestinal tuvo una media de $61,3 \pm 5,2$ puntos (de un máximo posible de 70), la sistémica tuvo una media de $24,8 \pm 8,8$ (de un máximo posible de 35), el emocional fue el más variable, con una media de $67,4 \pm 13,3$ (de un máximo posible de 84 ), y el dominio menos variable fue el social, cuya media fue de 32,6 \pm 3.6 (de un máximo posible de 35 ). Los valores de IBDQ32 no mostraron diferencias relevantes respecto al género (Figura 3A). Además, no se observaron diferencias relevantes en el puntaje total del IBDQ32 con respecto a la edad (Figura 3B), aunque los hombres presentaron valores más altos en la dimensión emocional (datos no mostrados). 


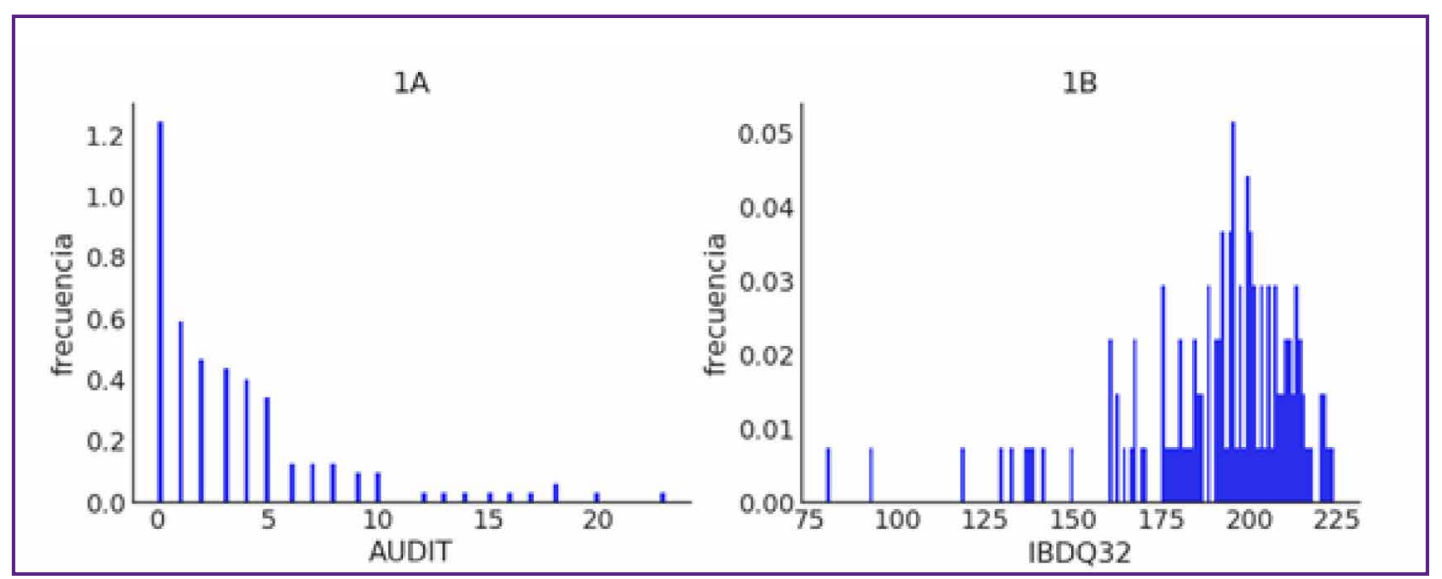

Figura 1. Histogramas; dado que AUDIT e IBDQ32 son variables discretas se usó un número de bins igual al de valores únicos en la muestra. Figura 1A. histograma de AUDIT. Figura 1B. histograma de IBDQ32

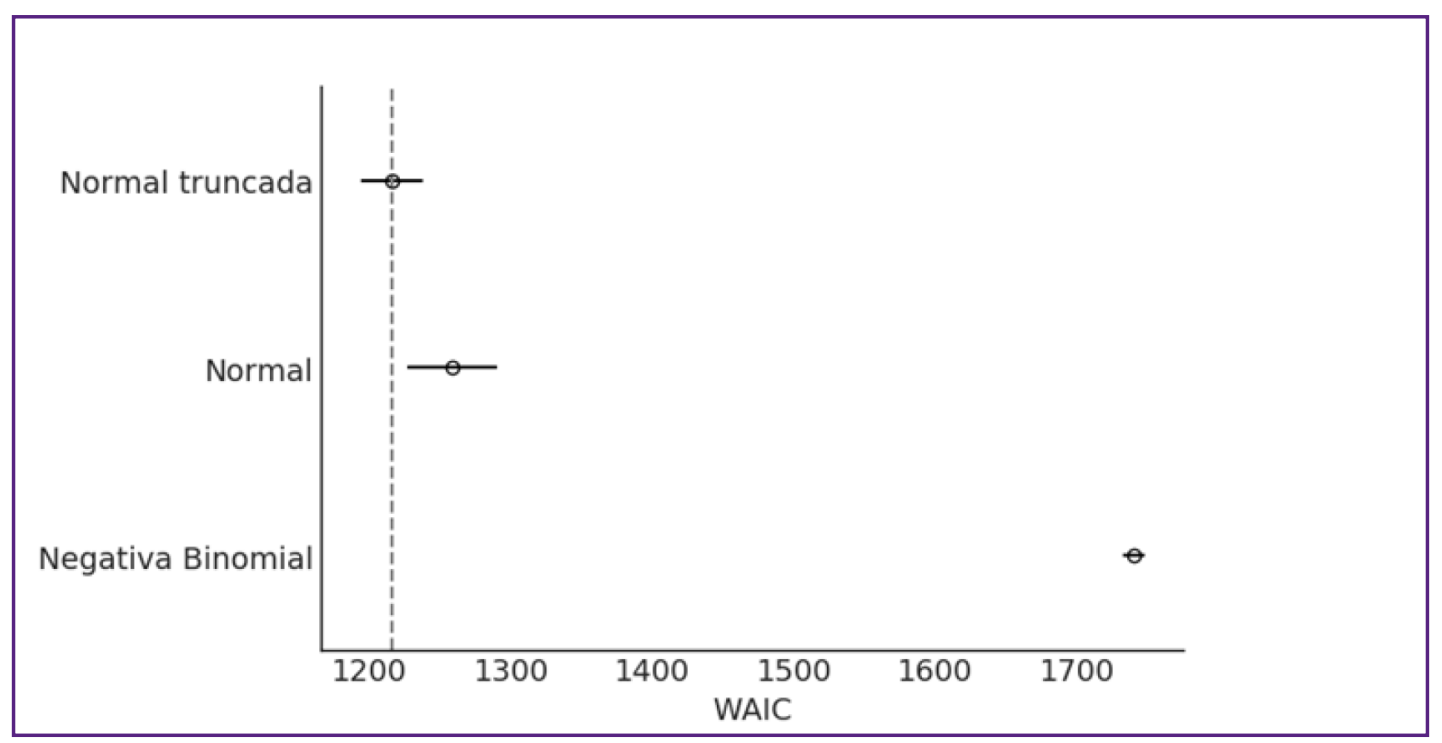

Figura 2. Gráfica de la evaluación de las distribuciones de IBDQ32 (normal, normal truncada y binomial negativa por WAIC)

Para lograr un análisis más completo de las diferencias de valores observados en el IBDQ32 de la muestra, se estimó la distribución del IBDQ32 en varones y se restó de la misma la distribución del IBDQ32 en mujeres, observando (Figura 4A) que las diferencias toman mayormente valores positivos, como lo indica su intervalo HPD (highest posterior density, es decir, el intervalo más corto posible que contiene un determinado porcentaje del total de la densidad de probabilidad, un concepto bayesiano análogo al intervalo de confianza 
frecuentista). Es decir, el conjunto de hombres tiene en promedio mayor IBDQ32 que el de mujeres, y el valor de la media de las diferencias (mean) es de 9,8 . El mismo razonamiento se debe usar para la Figura 4B, donde están involucrados los diferentes grupos etarios, 18 a 35, 35 a 60 y > 60. De estas figuras se puede observar que la mejor calidad de vida se localiza el segmento etario medio (de 35 a 60).

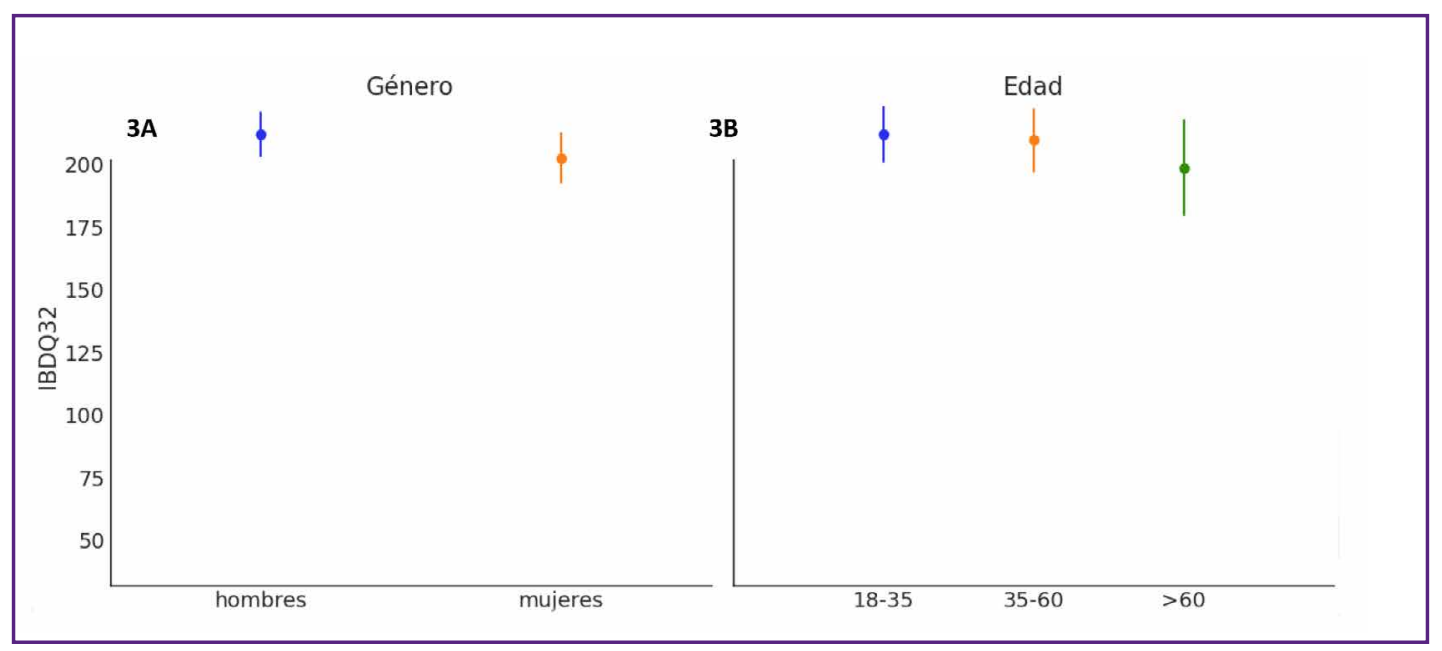

Figura 3. Medias del IBDQ32 discriminadas por sexo (3A) y por rango etario (3B)

En cuanto a la frecuencia alimentaria, se observa gran variabilidad de respuestas. Por ejemplo, solo el $63 \%$ de los encuestados llenó los campos correspondientes a lácteos, y el $81 \%$ los de verduras. En cuanto al consumo de carne, el $68 \%$ reportó algún consumo, y el $73 \%$ la consume al menos una vez a la semana. No todas las personas encuestadas reportan consumir alimentos que contengan cultivos andinos. Solo el $24,2 \%$ de las personas, alguno de ellos (quínoa, amaranto o papas andinas). Además, el 13,2\% incluye cultivos andinos en su dieta una sola vez a la semana, mientras que el $11 \%$ lo hace más de una vez a la semana.

Con el fin de investigar las relaciones existentes entre el patrón de consumo, la calidad de vida desde el punto de vista de la salud intestinal y la frecuencia de consumo de alimentos que contengan cultivos andinos, se realizó un análisis de componentes principales (Figura 5). Para este análisis se utilizó la implementación de PCA de Scikit-learn, que es un PCA probabilístico tal cual se describe en Probabilistic principal component analysis. (Tipping y Bishop, 1999) se utilizaron los parámetros por defecto y los datos fueron estandarizados antes de hacer el PCA. Se observa que el consumo de alcohol 
y la calidad de vida desde el punto de vista de la salud intestinal poseen una correlación negativa, es decir, patrones de alto consumo de alcohol tienden a asociarse con valores bajos de IBDQ32, y viceversa. Por otra parte, la calidad de vida se asocia positivamente con el consumo de papas andinas, mientras que el consumo de quínoa no muestra una tendencia clara de asociación con las otras variables.

$4 \mathrm{~A}$
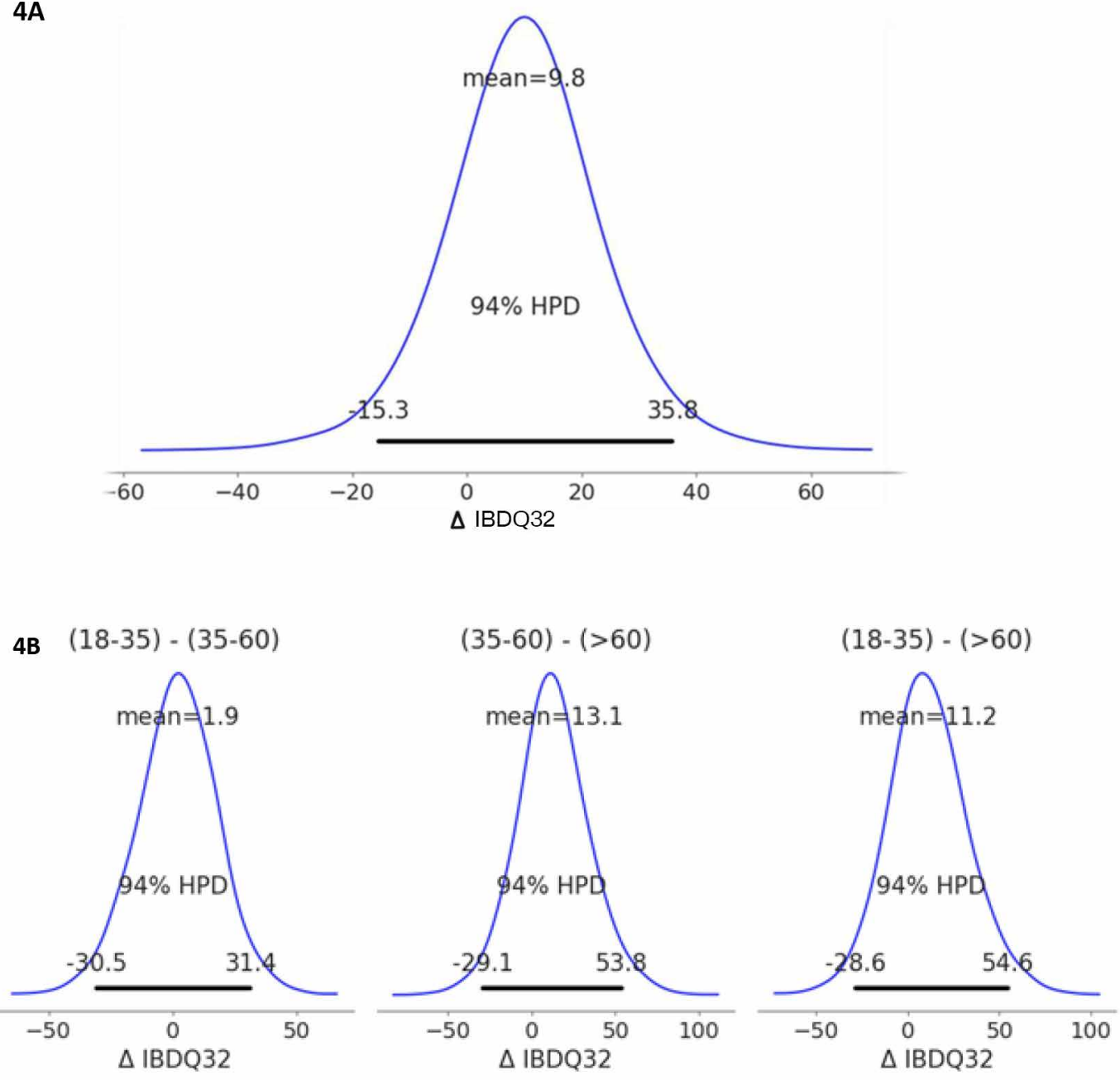

Figura 4. Valores de las diferencias de todos los registros de Audit, agrupados por sexo, varones - mujeres (4A) y entre los diferentes grupos etarios (4B) 


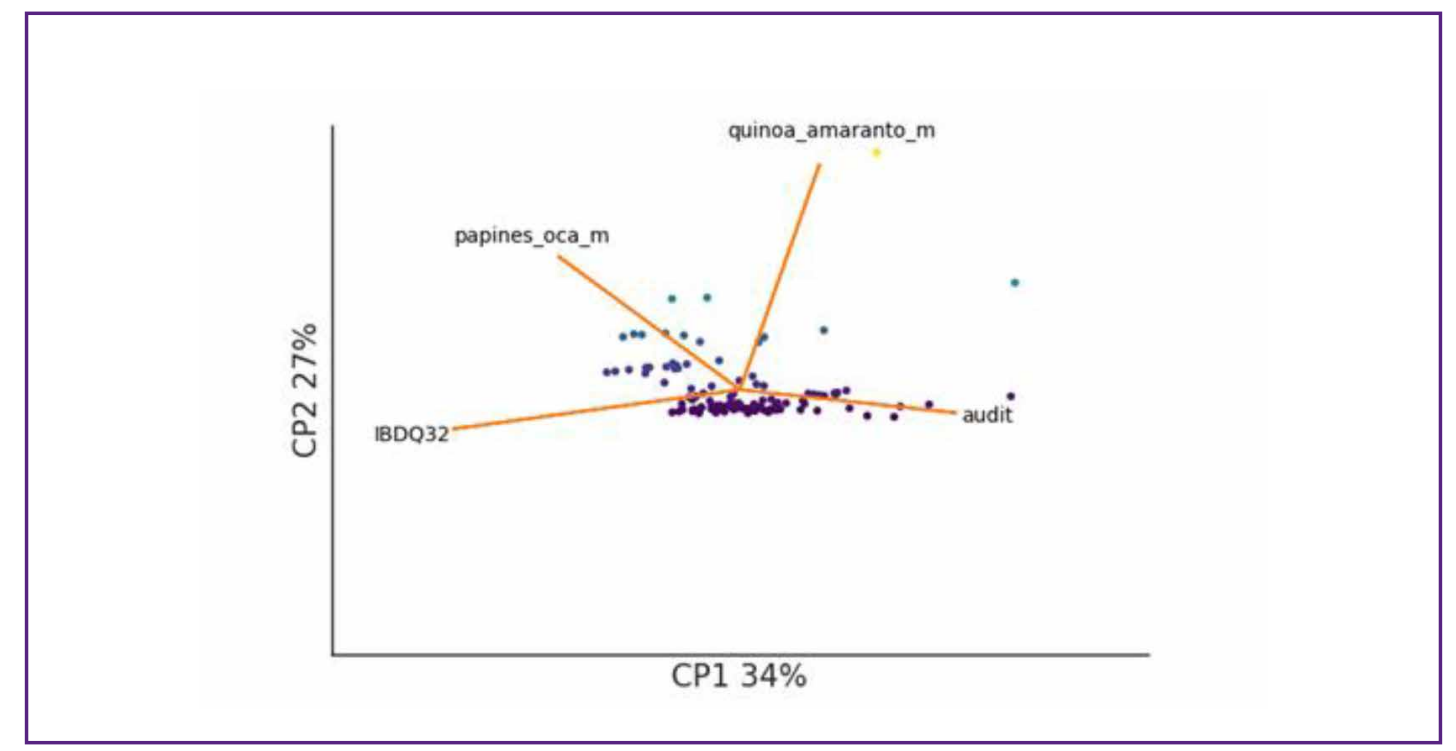

Figura 5. Análisis de componentes principales entre AUDIT, IBDQ32, consumo de papa andina (papines, oca), y consumo de quínoa y amaranto al mes

Referencias. Los puntos en el gráfico representan los valores puntuales de las muestras proyectadas en las dos dimensiones, Los colores más claros son valores más atípicos, los colores más oscuros son valores más típicos. La escala de colores usada se llama viridis (https://www.youtube.com/watch?v=XAoljeRJ3lU) y está construida exactamente para que el sistema perceptual humano intérprete cambios de color de acuerdo a los cambios en los datos, es decir tratando de introducir la mínima distorsión posible. Si bien la mayoría de los puntos tienen colores similares entre sí, unos pocos no. Eso es una característica de los datos, no un artefacto del gráfico. Es esperable que la mayoría de los puntos se vean muy parecidos, porque lo son. Agregar otra escala de colores (que no sea perceptualmente uniforme) o introducir símbolos introduciría distorsiones arbitrarias.

Se realizó una regresión lineal simple entre AUDIT e IBDQ32 asumiendo una distribución normal truncada de IBDQ32, como se determinó previamente usando WAIC (Figura 6). Se observa la misma tendencia que en el ACP: cuanto mayor es el consumo de alcohol, menor es la calidad de vida. 


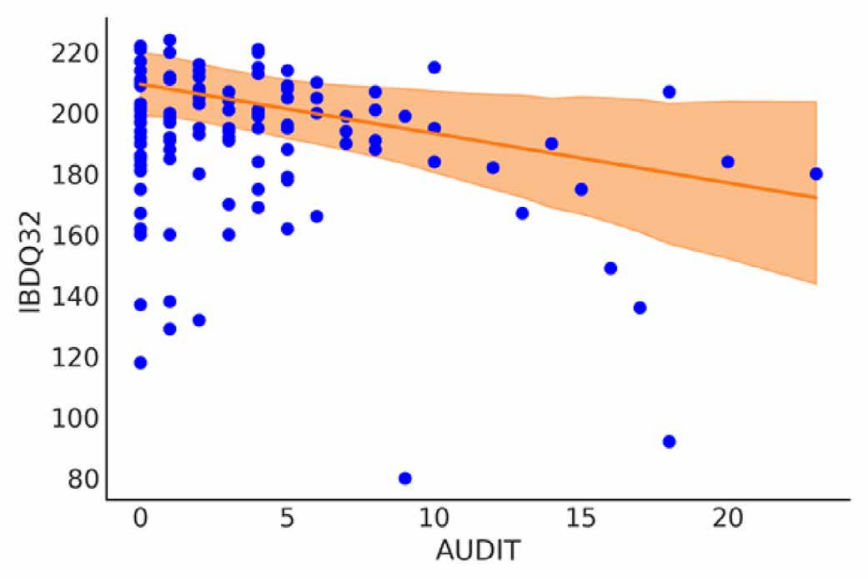

Figura 6. Regresión lineal entre AUDIT y IBDQ32. La línea naranja representa la tendencia central; el sombreado, el intervalo de credibilidad del $94 \%$

Finalmente, se realizaron varias regresiones lineales, todas ellas con IBDQ32 como variable respuesta (y asumiendo una distribución normal truncada para esta variable). Las diferentes combinaciones de variables predictoras se detallan en la Tabla 1. Se usó WAIC como criterio para comparar los distintos modelos. El modelo que mejor ajusta nuestra muestra es el que tiene como variables predictoras a los índices de AUDIT, la edad, el sexo, el índice de masa corporal y si consume alimentos andinos (quínoa, amaranto y papa andina) (Figura 7 y Tabla 1). Las diferencias entre valores de WAIC son muy pequeñas, indicando que todos los modelos evaluados tienen similar capacidad predictora.

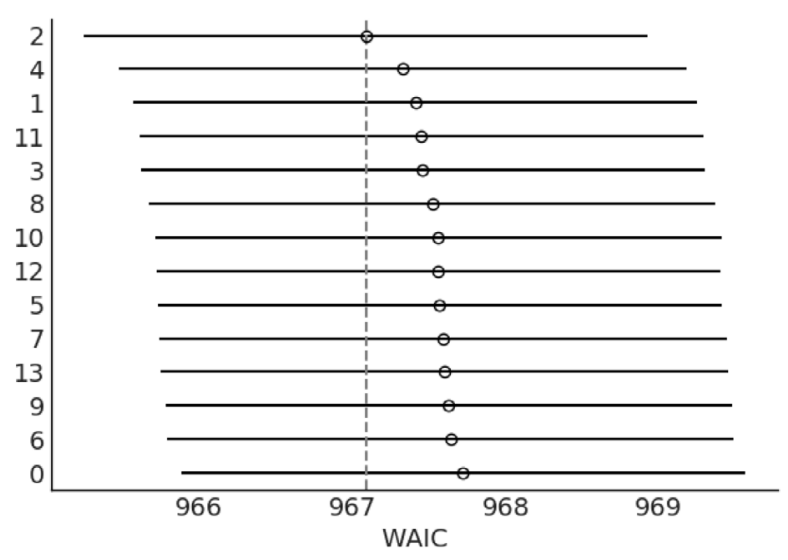

Figura 7. Comparación de modelos listados en la Tabla 1 
Tabla 1. Distintos modelos y sus variables para la selección WAIC de modelos

\begin{tabular}{|c|c|}
\hline Modelo & Variables \\
\hline 0 & IBDQ32, género, edad \\
\hline 1 & IBDQ32, género, edad, AUDIT \\
\hline 2 & IBDQ32, género, edad, AUDIT, consume alimentos andinos \\
\hline 3 & $\begin{array}{l}\text { IBDQ32, género, edad, AUDIT, consume alimentos andinos, índice de masa cor- } \\
\text { poral }\end{array}$ \\
\hline 4 & $\begin{array}{l}\text { IBDQ32, género, edad, AUDIT, consume alimentos andinos, índice de masa cor- } \\
\text { poral, sumatoria de lácteos consumidos por semana }\end{array}$ \\
\hline 5 & $\begin{array}{l}\text { IBDQ32, género, edad, AUDIT, índice de masa corporal, sumatoria de hidratos de } \\
\text { carbono consumidos por semana }\end{array}$ \\
\hline 6 & $\begin{array}{l}\text { IBDQ32, género, edad, AUDIT, índice de masa corporal, sumatoria de verduras } \\
\text { consumidas por semana }\end{array}$ \\
\hline 7 & $\begin{array}{l}\text { IBDQ32, género, edad, AUDIT, índice de masa corporal, sumatoria de carnes con- } \\
\text { sumidas por semana }\end{array}$ \\
\hline 8 & $\begin{array}{l}\text { IBDQ32, género, edad, AUDIT, índice de masa corporal, sumatoria de cereales y } \\
\text { legumbres consumidas por semana }\end{array}$ \\
\hline 9 & $\begin{array}{l}\text { IBDQ32, género, edad, AUDIT, índice de masa corporal, sumatoria de frutas con- } \\
\text { sumidas por semana }\end{array}$ \\
\hline 10 & $\begin{array}{l}\text { IBDQ32, género, edad, AUDIT, índice de masa corporal, sumatoria de golosinas } \\
\text { consumidas por semana }\end{array}$ \\
\hline 11 & $\begin{array}{l}\text { IBDQ32, género, edad, AUDIT, índice de masa corporal, sumatoria de bebidas sin } \\
\text { alcohol consumidas por semana }\end{array}$ \\
\hline 12 & $\begin{array}{l}\text { IBDQ32, género, edad, AUDIT, índice de masa corporal, sumatoria de alimentos } \\
\text { andinos consumidos por semana }\end{array}$ \\
\hline 13 & $\begin{array}{l}\text { IBDQ32, género, edad, AUDIT, índice de masa corporal, sumatoria de alimentos } \\
\text { andinos consumidos por semana }\end{array}$ \\
\hline
\end{tabular}

\section{Discusión}

En este trabajo se utilizaron diferentes encuestas con el objetivo de encontrar relaciones entre el patrón de consumo de bebidas alcohólicas, la calidad de vida desde el punto de vista de la salud intestinal y la frecuencia de consumo de algunos alimentos. Existen numerosos estudios sobre la relación entre el consumo de alcohol y la calidad de vida (Parackal y Parackal, 2017) y trabajos sobre 
la alimentación y la calidad de vida en pacientes enfermos (Pasternack et al., 2015), pero este es el primer estudio en el que se analizan las tres relaciones.

Los puntajes de AUDIT no son normales, con una alta cantidad de personas que reportan un consumo bajo de alcohol. No existen estudios a gran escala en los que se utilice el AUDIT para analizar los patrones de consumo de alcohol en la población de Jujuy. Solo existen un par de estudios en poblaciones muy acotadas, como pueden ser las personas que llegan a la sala de emergencia (Alderete y Bianchini, 2008) o estudiantes de escuelas secundarias (Alderete et al., 2008), en las que se usó una versión acortada del AUDIT, llamada AUDIT-C. En estos estudios se encontraron patrones de consumo similares al nuestro. Comparando con datos obtenidos a nivel nacional (Blake, A., 2011), los valores de consumo de alcohol observados en este estudio son ligeramente mayores (esto es, el 62,9\% de los varones y el $38 \%$ de las mujeres consumieron alcohol al menos una vez en el último mes, según la encuesta nacional, mientras que en este estudio se observó que el $69,4 \%$ de varones y el $63 \%$ de mujeres consumen alcohol al menos una vez por mes). Nuestro análisis muestra valores mayores probablemente debido a factores socioculturales, y a que nuestro muestreo tiene un alto porcentaje de jóvenes (ambos sondeos coinciden en que la edad de mayor consumo es la adolescencia).

En un estudio realizado en España (Cortés et al., 2016) se evaluó la salud intestinal de cien pacientes con enfermedades intestinales (enfermedad de Crohn, colitis ulcerosa), llegando a resultados algo menores a los nuestros: los varones tuvieron una media de $180( \pm 31,4)$ contra 200,6 \pm 15.3 de nuestro estudio; las mujeres presentaron un índice mucho menor, 152,4 $\pm 43,3$ contra $184,2 \pm 26,5$. Es probable que estos valores menores se deban a que en el estudio español se entrevistaron personas diagnosticadas con enfermedades inflamatorias intestinales; sin embargo, las diferencias entre hombres y mujeres también son estadísticamente significativas en este estudio previamente mencionado (Cortés et al., 2016).

En este trabajo se analizó la frecuencia de consumo de diferentes grupos de alimentos, pero se tuvo especial interés en el consumo de aquellos de origen andino, debido a que este tipo de alimentos es producido en la región donde se lleva a cabo el estudio; además, tiene una composición que los hace buenos candidatos para influir en la salud intestinal (Repo-Carrasco-Valencia, s.f.).

Al analizar las relaciones entre las diferentes variables consideradas en este trabajo, se observó una relación inversa existente entre valores de AUDIT e IBDQ32 (Figuras 5 y 6), indicando que a mayor consumo de alcohol se produce un empeoramiento de la calidad de vida desde el punto de vista de la salud intestinal. Esta es la relación que se esperaba dada la cantidad de ob- 
servaciones realizadas en humanos (Rajendram y Preedy, 2005) y en modelos animales (Purohit et al., 2008) en las que se demuestra que el alcohol produce daños en la barrera epitelial intestinal. Además, no se observan relaciones de importancia, tanto en el ACP, las correlaciones individuales o las regresiones múltiples, que involucren a los alimentos andinos, u otros grupos de alimentos en el bienestar intestinal. Los valores que mejor explican esta distribución son los índices de AUDIT, la edad, el sexo, el índice de masa corporal, y si consume alimentos andinos (quínoa, amaranto y papa andina).

Es importante notar que los resultados de este trabajo son preliminares. Probablemente una muestra más grande permitiría aumentar el nivel de resolución del análisis, permitiendo encontrar efectos pequeños de los alimentos en relación a la salud intestinal y los efectos del alcohol sobre la misma.

Conflicto de intereses: Ninguno para declarar. 


\section{Referencias bibliográficas}

Alderete, E., y Bianchini, P. (2008). Alcohol consumption in the emergency room. Medicina, 68(1), 31-36.

Alderete, E.; Kaplan, C. P.; Nah, G., y Pérez-Stable, E. J. (2008). Problemas relacionados con el consumo de alcohol en jóvenes de la provincia de Jujuy, Argentina. Salud Pública de México, 50(4), 300-307. DOI: https:// doi.org/10.1590/S0036-36342008000400008

ANDERSON, J. M., y Van Itallie, C. M. (2009). Physiology and function of the tight junction. Cold Spring Harbor Perspectives in Biology, 1(2), a002584. https://doi org/10.1101/cshperspect.a002584

BABOR, T, H. B. J.; Saunders, J., y Monteiro, M. (2001). Audit. The alcohol use disorders identification test. Who/MSD/MSB.

BLAKE, A. (2011). Estado de situación. Consumo problemático de alcohol en Argentina. Dirección Nacional de Salud Mental y Adicciones.

CASELLAS, F., López-Vivancos, J.; Badia, X.; Vilaseca, J., y Malagelada, J. R. (2001). Influence of inflammatory bowel disease on different dimensions of quality of life. European Journal of Gastroenterology \& Hepatology, 13(5), 567-572.

CASELLAS, F.; López-Vivancos, J.; Casado, A., y Malagelada, J. R. (2002). Factors affecting health related quality of life of patients with inflammatory bowel disease. Quality of Life Research: An International Journal of Quality of Life Aspects of Treatment, Care and Rehabilitation, 11(8), 775-781.

CORTÉS, R. L.; Fernández, B. M.; Montoro, C. H.; Hernández, P. E.; Aznarez, A. C. S., y Gutiérrez, C. R. (2016). Quality of life in patients with inflammatory bowel diseases. An. Sist. Sanit. Navar., 39, 9.

GUYATT, G. H.; Feeny, D. H., y Patrick, D. L. (1993). Measuring health-related quality of life. Annals of Internal Medicine, 118, 622-629.

HUNTER, J. D. (2007). Matplotlib: A 2D graphics environment. Computing in Science \& Engineering, 9(3), 90-95. DOI: https://doi. org/10.1109/MCSE.2007.55

Keshavarzian, A.; Holmes, E. W.; Patel, M.; Iber, F.; Fields, J. Z., y Pethkar, S. (1999). Leaky gut in alcoholic cirrhosis: A possible mechanism for alcohol-induced liver damage. The American Journal of Gastroenterology, 94(1), 200-207.

KUMAR, R.; Carroll, C.; Hartikainen, A., y Martin, O. (2019). ArviZ a unified library for exploratory analysis of Bayesian models in Python. Journal of Open Source Software, 4(33), 1143. DOI: https://doi.org/10.21105/ joss.01143

MARTIN-Moreno, J. M.; Boyle, P.; Gorgojo, L.; Maisonneuve, P.; Fernandez-Rodriguez, J. C.; Salvini, S., y Willett, W. C. (1993). Development and validation of a food frequency questionnaire in Spain. International Journal of Epidemiology, 22(3), 512-519.

MASACHS, M.; Casellas, F., y Malagelada, J. R. (2007). Traducción, adaptación y validación al español del cuestionario de calidad de vida de 32 ítems (IBDQ-32) de la enfermedad inflamatoria intestinal. Revista Española de Enfermedades digestivas, 99(9), 511-519.

MCKINNEY, W. (2011). Pandas: A Foundational Python Library for Data Analysis and Statistics.

MedinA, A. L.; Arévalo, N. M.; Beltrán, S. D.; Chavarro, Y. L.; Herazo, E., y Campo-Arias, 
A. (2015). Consistencia interna y estructura interna del cuestionario AUDIT en amerindios. 16(28), 10.

PARACKAL, M., y Parackal, S. (2017). Implication of alcohol consumption on aggregate wellbeing. Perspectives in Public Health, 137(4), 220-226. DOI: https://doi. org/10.1177/1757913916669538

PASCUAL, S.; Martínez, J., y Pérez-Mateo, M. (2001). La barrera intestinal: Trastornos funcionales en enfermedades digestivas y extradigestivas. Gastroenterología y Hepatología, 24(5), 256-267. DOI: https://doi. org/10.1016/50210-5705(01)70167-7

PASTERNACK, C.; Kaukinen, K.; Kurppa, K.; Mäki, M.; Collin, P.; Reunala, T.; Huhtala, H., y Salmi, T. (2015). Quality of life and gastrointestinal symptoms in long-term treated dermatitis herpetiformis patients: A cross-sectional study in Finland. American Journal of Clinical Dermatology, 16(6), 545-552. DOI: https://doi.org/10.1007/s40257-015-0149-1

Pedregosa, F.; Varoquaux, G.; Gramfort, A.; Michel, V.; Thirion, B.; Grisel, O.; Blondel, M.; Prettenhofer, P.; Weiss, R.; Dubourg, V.; Vanderplas, J.; Passos, A., y Cournapeau, D. (2011). Scikit-learn: Machine learning in Python. MACHINE LEARNING IN PYTHON, 6. PUROHIT, V.; Bode, J. C.; Bode, C.; Brenner, D. A.; Choudhry, M. A.; Hamilton, F.; Kang, Y. J.; Keshavarzian, A.; Rao, R.; Sartor, R. B.; Swanson, C., y Turner, J. R. (2008). Alcohol, intestinal bacterial growth, intestinal permeability to endotoxin, and medical consequences: Summary of a symposium. Alcohol, 42(5), 349-361. DOI: https://doi.org/10.1016/j.alcohol.2008.03.131

QUIDEAU, S.; Deffieux, D.; Douat-Casassus, C., y Pouységu, L. (2011). Plant polyphenols:
Chemical properties, biological activities, and synthesis. Angewandte Chemie (International Ed. in English), 50(3), 586-621. DOI: https://doi.org/10.1002/anie.201000044 RAJENDRAM, R., y Preedy, V. R. (2005). Effect of alcohol consumption on the gut. Digestive Diseases, 23(3-4), 214-221. DOI: https://doi. org/10.1159/000090168

RePo-CARRASCO-VAlenCIA, R. (s.f.). Andean Indigenous Food Crops: Nutritional Value and Bioactive Compounds. 189.

SAlVATIER, J.; Wiecki, T. V., y Fonnesbeck, C. (2016). Probabilistic programming in Python using PyMC3. PeerJ Computer Science, 2, e55. DOI: https://doi.org/10.7717/ peerj-cs. 55

Salvo Romero, E.; Alonso Cotoner, C.; Pardo Camacho, C.; Casado Bedmar, M., y Vicario, M. (2015). The intestinal barrier function and its involvement in digestive disease. Revista Española de Enfermedades Digestivas: Órgano Oficial de la Sociedad Española de Patología Digestiva, 107(11), 686-696.

TIPPING, M. E., y Bishop, C. M. (1999). Probabilistic principal component analysis. Neural Computing Research Group.

VergaRA, M.; Casellas, F.; Badia, X., y Malagelada, J. R. (2002). Assessing the quality of life of household members of patients with inflammatory bowel disease: Development and validation of a specific questionnaire. The American Journal of Gastroenterology, 97(6), 1429-1437.

Watanabe, S. (2013). A Widely Applicable Bayesian Information Criterion. 31.

WILLETT, W. C.; Sampson, L.; Stampfer, M. J.; Rosner, B.; Bain, C.; Witschi, J.; Hennekens, C. H., y Speizer, F. E. (1985). Reproducibility 
and validity of a semiquantitative food frequency questionnaire. American Journal of Epidemiology, 122(1), 51-65. DOI: https:// doi.org/10.1093/oxfordjournals.aje.a114086 YANG, G.; Bibi, S.; Du, M.; Suzuki, T., y Zhu, M.J. (2017). Regulation of the intestinal tight junction by natural polyphenols: A mechanistic perspective. Critical Reviews in Food Science and Nutrition, 57(18), 3830-3839. DOI: https://doi.org/10.1080/10408398.2016. $\underline{1152230}$ 